\title{
Products of Prime Powers in Binary Recurrence Sequences Part II: The Elliptic Case, with an Application to a Mixed Quadratic-Exponential Equation
}

\author{
By B. M. M. de Weger
}

\begin{abstract}
In Part I the diophantine equation $G_{n}=w p_{1}^{m_{1}} \cdots p_{t}^{m_{t}}$ was studied, where $\left\{G_{n}\right\}_{n=0}^{\infty}$ is a linear binary recurrence sequence with positive discriminant. In this second part we extend this to negative discriminants. We use the $p$-adic and complex Gelfond-Baker theory to find explicit upper bounds for the solutions of the equation. We give algorithms to reduce those bounds, based on diophantine approximation techniques. Thus we have a method to solve the equation completely for arbitrary values of the parameters. We give an application to a quadratic-exponential equation.
\end{abstract}

\section{Introduction and Preliminaries.}

6A. Introduction. It is assumed that the reader is familiar with Part I of this paper (Pethö and de Weger [4]). We adopt notations and assumptions from Part I without further reference.

In Part I we studied Eq. (1.1):

$$
G_{n}=w p_{1}^{m_{1}} \cdots p_{t}^{m_{t}}
$$

for $\Delta>0$. The $p$-adic Gelfond-Baker theory, together with a trivial observation on the exponential growth of $\left|G_{n}\right|$, provided us with upper bounds for the solutions. In the case $\Delta<0$, which is our present topic, the situation is essentially more complicated. The $p$-adic behavior of $G_{n}$ does not depend on the sign of the discriminant. But in the case $\Delta<0$, the growth of $\left|G_{n}\right|$ is not as nice as in the case $\Delta>0$. However, information on its growth can be obtained from the complex Gelfond-Baker theory. The fact that Eq. (1.1) has only finitely many solutions was shown by Mahler [3].

Section 7 is devoted to the complex arguments. In it we solve the diophantine inequality $\left|G_{n}\right| \leqslant v$ for a fixed $v$. An upper bound for $n$ is given that has particularly good dependence on $v$. We present algorithms to reduce this upper bound, so that the inequality can be solved completely in any practical case. These algorithms are not new; they come essentially from Baker and Davenport [1] and Cijsouw, Korlaar, and Tijdeman (appendix to Stroeker and Tijdeman [5]).

Received December 13, 1985.

1980 Mathematics Subject Classification (1985 Revision). Primary 11B37, 11D61, 11J87, 11Y50. 
In Subsection $8 \mathrm{~A}$ we combine the results of Sections 3 and 7 to obtain explicit upper bounds for (1.1). In Subsection $8 \mathrm{~B}$ an algorithm is presented to reduce these upper bounds. It is a combination of the algorithms of Sections 4 and 7. We give an example in Subsection 8C. Finally, in Section 9 we present an application to a certain type of mixed quadratic-exponential diophantine equation.

6B. Preliminaries. Let in the sequel $\Delta<0$. Since $\alpha / \beta$ is not a root of unity, $B \geqslant 2$. Since $(\alpha, \beta)$ and $(\lambda, \mu)$ are pairs of complex conjugates, $|\alpha|=|\beta|$ and $|\lambda|=|\mu|$. Thus $L=\log \max \left(|e D|^{1 / 4},|\alpha \lambda \sqrt{D}|\right)$. Lemmas 3.2, 4.2, and 4.3 hold also for $\Delta<0$.

As in the case $\Delta>0$, we have to exclude the case where only finitely many $p_{i}$-adic digits of $\theta_{i}$ are nonzero. Let $\rho=\frac{1}{2}(1+\sqrt{-3})$.

LEMMA 6.1. If only finitely many $p_{i}$-adic digits $u_{i, l}$ of $\theta_{i}$ are nonzero, then $\theta_{i}=0$, and $G_{n}= \pm R_{n}, \kappa S_{n}, \kappa T_{n}$ or $\kappa U_{n}$, where $\kappa \in \mathbb{Q}$, and

$$
\begin{aligned}
R_{n} & =\left(\alpha^{n}-\beta^{n}\right) /(\alpha-\beta), \quad S_{n}=\alpha^{n}+\beta^{n}, \\
T_{n} & =(1 \pm \sqrt{-1}) \alpha^{n}+(1 \mp \sqrt{-1}) \beta^{n}, \\
U_{n} & =(1 \pm \omega) \alpha^{n}+(1 \pm \bar{\omega}) \beta^{n}, \quad \omega=\rho \text { or } \bar{\rho} .
\end{aligned}
$$

The case $G_{n}=\kappa T_{n}$ can occur only if $d=-1$, and $G_{n}=\kappa U_{n}$ only if $d=-3$.

Proof. As in the proof of Lemma 4.4, $\theta_{i}=r \in \mathbb{Z}$, and $(\beta / \alpha)^{r}(\mu / \lambda)=\eta$ is a root of unity. Then $\eta \lambda \alpha^{r}=\mu \beta^{r}$, hence

$$
G_{n}=\lambda \alpha^{r}\left(\alpha^{n-r}+\eta \beta^{n-r}\right) .
$$

Recall that $B=\alpha \beta \geqslant 2$. Notice that

$$
G_{0} B\left(\eta \alpha^{r-1}+\beta^{r-1}\right)=G_{1}\left(\eta \alpha^{r}+\beta^{r}\right) .
$$

By $\left(B, G_{1}\right)=1$, it follows that $\alpha \beta \mid \eta \alpha^{r}+\beta^{r}$. By $(A, B)=1$, we have $(\alpha, \beta)=(1)$, and from $\alpha \mid \beta^{r}$ it then follows that $\theta_{i}=r=0$. So $G_{0}=\lambda(1+\eta) \in \mathbb{Z}$. Then $\lambda=$ $\kappa(1+\bar{\eta})$ for some $\kappa \in \mathbb{Q}$. Choose $\kappa$ such that $G_{0}, G_{1} \in \mathbb{Z}$ and $\left(G_{0}, G_{1}\right)=1$. Now the result follows easily, since for $\eta$ there are only the possibilities \pm 1 , and $\pm \sqrt{-1}$ if $d=-1$, and $\pm \rho, \pm \bar{\rho}$ if $d=-3$.

In the cases of Lemma 6.1, Eq. (1.1) can be treated as follows. The smallest index $n=g\left(m p^{l}\right)$ such that $m p^{l} \mid G_{n}$ grows exponentially with $l$. Also $G_{n}$ grows exponentially with $n$ (see Theorem 7.2). Hence $G_{g\left(m p^{\prime}\right)}$ grows double exponentially with $l$. It follows that $w p_{1}^{m_{1}} \cdots p_{t}^{m_{t}}$ cannot keep up with $G_{g\left(w p_{1}^{m_{1}} \ldots p_{t}^{\left.m_{t}\right)}\right.}$. So, if $m_{1}, \ldots, m_{t}$ are large enough, there is a prime $q$ such that $q \mid G_{g\left(w p_{1}^{m_{1}} \ldots p_{t}^{\left.m_{t}\right)}\right.}$, but $q+w p_{1}^{m_{1}} \cdots p_{t}^{m_{t}}$. Now the special properties of the sequences $R_{n}, S_{n}, T_{n}$, and $U_{n}$ can be employed to prove that $q \mid G_{n}$ whenever $w p_{1}^{m_{1}} \cdots p_{t}^{m_{t}} \mid G_{n}$. We illustrate this with an example.

Let $A=5, B=13, G_{0}=G_{1}=1$. Then $\Delta=-27, \alpha=1+3 \rho, \lambda=(1+\rho) / 3$. We solve $G_{n}= \pm 2^{m}$. The sequence $G_{n}=\lambda \alpha^{n}+\bar{\lambda} \bar{\alpha}^{n}$ is related to the sequence $H_{n}=\bar{\lambda} \alpha^{n}+\lambda \bar{\alpha}^{n}$. In fact, we have $G_{n} H_{n} R_{n}=R_{3 n} / 3$. Since $R_{n}$ has nice divisibility properties, we thus have information on the prime divisors of $G_{n}$ and $H_{n}$. We find:

\begin{tabular}{l|rrrrrrrrr}
$n$ & 0 & 1 & 2 & 3 & 4 & 5 & 6 & 7 & 8 \\
\hline$G_{n}$ & 1 & 1 & -8 & -53 & -161 & -116 & 1513 & 9073 & 25696 \\
$H_{n}$ & 1 & 4 & 7 & -17 & -176 & -659 & -1007 & 3532 & 30751 \\
$R_{n}$ & 0 & 1 & 5 & 12 & -5 & -181 & -840 & -1847 & 1685
\end{tabular}


Now $G_{n} \equiv 0(\bmod 16)$ if and only if $n \equiv 8(\bmod 12), H_{n} \equiv 0(\bmod 16)$ if and only if $n \equiv 4(\bmod 12)$, and $R_{n} \equiv 0(\bmod 16)$ if and only if $n \equiv 0(\bmod 12)$. Further, $G_{4} H_{4} R_{4}=R_{12} / 3=-2^{4} \cdot 5 \cdot 7 \cdot 11 \cdot 23$, and it follows that $2^{4} \cdot 7 \cdot 11 \cdot 23 \mid G_{n} H_{n}$ for all $n \equiv 0(\bmod 4)$. In fact, $11 \mid G_{n}$ whenever $16 \mid G_{n}$. Thus $G_{n}= \pm 2^{m}$ implies $m \leqslant 3$. In the next section we show how to solve $\left|G_{n}\right| \leqslant 8$.

Another way to treat (1.1) in the case $\theta_{i}=0$ is the following. By Lemma 4.2, $m_{i} \leqslant g_{i}+1+\operatorname{ord}_{p_{i}}(n)$. Hence,

$$
\left|G_{n}\right|=|w| p_{1}^{m_{1}} \cdots p_{t}^{m_{t}} \leqslant v_{0} n
$$

for some constant $v_{0}$. Only minor changes in the arguments of Section 7 suffice to deal with this inequality, instead of $\left|G_{n}\right| \leqslant v$.

7. The Growth of the Recurrence Sequence.

7A. Application of a Theorem of Waldschmidt. In this subsection we study the diophantine inequality

$$
\left|G_{n}\right| \leqslant v
$$

for a fixed $v \in \mathbb{R}, v \geqslant 1$. We apply a result of Waldschmidt [6] from the complex Gelfond-Baker theory, which gives an upper bound for $n$ that is particularly good in $v$. See also Kiss [2].

Let $a_{0}$ for $\xi \in \mathbb{Q}(\sqrt{\Delta})$ be the leading coefficient of its minimal polynomial. We define the height of $\xi$ by

$$
h(\xi)=\frac{1}{2} \log a_{0}+\log \max (1,|\xi|),
$$

in accordance with Waldschmidt's height function (cf. [6, p. 259]). Let $\alpha_{1}, \ldots, \alpha_{n} \in$ $\mathbb{Q}(\sqrt{\Delta}), b_{1}, \ldots, b_{n} \in \mathbb{Z}$. Put

$$
\Lambda=b_{1} \log \alpha_{1}+\cdots+b_{n} \log \alpha_{n},
$$

where $\log$ denotes the principal value of the complex logarithm, i.e., $-\pi<\operatorname{Im} \log z$ $\leqslant \pi$. Assume $\Lambda \neq 0$. Let $V_{1}, \ldots, V_{n}$ be real numbers with $\frac{1}{2} \leqslant V_{1} \leqslant \cdots \leqslant V_{n}$, and $V_{i} \geqslant \max \left\{h\left(\alpha_{i}\right), \frac{1}{2}\left|\log \alpha_{i}\right|\right\}(i=1, \ldots, n)$. Put $W=\max _{1 \leqslant i \leqslant n} \log \left|b_{i}\right|$. Define $V_{i}^{+}=$ $\max \left(1, V_{i}\right)$ for $i=n-1, n$. Put

$$
C_{4}=2^{9 n+53} n^{2 n} V_{1} \cdots V_{n} \log \left(2 e V_{n-1}^{+}\right), \quad C_{5}=C_{4} \log \left(2 e V_{n}^{+}\right) .
$$

THEOREM 7.1 (WALDSCHMIDT). With the above definitions,

$$
|\Lambda|>\exp \left\{-\left(C_{4} W+C_{5}\right)\right\} \text {. }
$$

We apply this to (7.1) as follows. Let

$$
\begin{aligned}
& E=-\lambda \mu \Delta, \\
& U_{2}=\frac{1}{2} \max (\pi, \log B), \quad U_{3}=\frac{1}{2} \max (\pi, \log E), \\
& U_{2}^{+}=\min \left(U_{2}, U_{3}\right), \quad U_{3}^{+}=\max \left(U_{2}, U_{3}\right), \\
& C_{4}^{\prime}=2^{79} 3^{6} U_{2} U_{3} \log \left(2 e U_{2}^{+}\right), \quad C_{5}^{\prime}=C_{4}^{\prime} \log \left(4 e U_{3}^{+}\right), \\
& C_{6}=\left(\log (\pi / 2|\mu|)+C_{5}^{\prime}+C_{4}^{\prime} \log \left(4 C_{4}^{\prime} / \log B\right)\right) \times 4 / \log B .
\end{aligned}
$$


THEOREM 7.2. Let $v \in \mathbb{R}, v \geqslant 1$. Then all solutions $n \geqslant 0$ of (7.1) satisfy

$$
n<C_{6}+\frac{4}{\log B} \log \max \left(v, 2\left|G_{0} \mu \sqrt{\Delta}\right|\right) \text {. }
$$

Remark. Notice that $C_{6}$ does not depend on $v$.

Proof. By $\Delta<0$, both $(\alpha, \beta)$ and $(\lambda, \mu)$ are pairs of complex conjugates. Hence $|\alpha|=|\beta|=B^{1 / 2} \geqslant \sqrt{2}$. We have from (7.1)

$$
\left|\left(\frac{-\lambda}{\mu}\right)\left(\frac{\alpha}{\beta}\right)^{n}-1\right| \leqslant \frac{v}{|\mu|} B^{-n / 2}
$$

We may assume $n \geqslant 2$. Let $-\lambda / \mu=e^{2 \pi i \psi}, \alpha / \beta=e^{2 \pi i \phi}$, with $-\frac{1}{2}<\phi \leqslant \frac{1}{2},-\frac{1}{2}$ $<\psi \leqslant \frac{1}{2}$. Let $k_{0}, k_{1} \in \mathbb{Z}$ be such that $\left|j \psi+n \phi+k_{j}\right| \leqslant \frac{1}{2}$. Then $\left|k_{j}\right| \leqslant 1+\frac{1}{2} n \leqslant n$ $(j=0,1)$. Put

$$
\Lambda_{j}=2 \pi i\left(j \psi+n \phi+k_{j}\right)=j \log \left(\frac{-\lambda}{\mu}\right)+n \log \left(\frac{\alpha}{\beta}\right)+2 k_{j} \log (-1)
$$

for $j=0,1$. It is an easy exercise to show that $|x| \leqslant \frac{1}{4}\left|e^{2 \pi i x}-1\right|$ holds for all $x \in \mathbb{R}$ with $|x| \leqslant \frac{1}{2}$. Now, from (7.2) we have an upper bound for $\left|\Lambda_{1}\right|$ :

$$
\begin{aligned}
\left|\Lambda_{1}\right| & =2 \pi\left|\psi+n \phi+k_{1}\right| \leqslant \frac{\pi}{2}\left|e^{2 \pi i\left(\psi+n \phi+k_{1}\right)}-1\right| \\
& =\frac{\pi}{2}\left|\left(\frac{-\lambda}{\mu}\right)\left(\frac{\alpha}{\beta}\right)^{n}-1\right| \leqslant \frac{\pi}{2|\mu|} v B^{-n / 2} .
\end{aligned}
$$

It may happen that $\Lambda_{1}=0$. In that case, $\psi+n \phi \in \mathbb{Z}$, hence $-(\lambda / \mu)(\alpha / \beta)^{n}=1$, and it follows that $G_{n}=\lambda \alpha^{n}+\mu \beta^{n}=0$. Kiss [2] showed that this implies $\left|R_{n}\right| \leqslant$ $2\left|G_{0}\right|$, where $R_{n}=\left(\alpha^{n}-\beta^{n}\right) /(\alpha-\beta)$. From this, Kiss derived an upper bound for $n$. We shall follow his argument, but we apply another, sharper result from the Gelfond-Baker theory than Kiss. Notice that, by $|\beta|=B^{1 / 2}$,

$$
2\left|G_{0}\right| \geqslant\left|R_{n}\right|=\frac{B^{n / 2}}{\sqrt{|\Delta|}}\left|\left(\frac{\alpha}{\beta}\right)^{n}-1\right| \geqslant \frac{4 B^{n / 2}}{\sqrt{|\Delta|}}\left|\phi n+k_{0}\right|=\frac{2 B^{n / 2}}{\pi \sqrt{|\Delta|}}\left|\Lambda_{0}\right| .
$$

Now $\Lambda_{0} \neq 0$, since by $n \geqslant 2$ the contrary would imply $\phi \in \mathbb{Q}$, which is impossible, since $\alpha / \beta$ is not a root of unity. Thus, take $j=1$ if $\Lambda_{1} \neq 0$, and $j=0$ otherwise. Then $\Lambda_{j} \neq 0$, and

$$
\left|\Lambda_{j}\right| \leqslant \frac{\pi}{2|\mu|} \max \left(v, 2\left|G_{0} \mu \sqrt{\Delta}\right|\right) B^{-n / 2} .
$$

From Theorem 7.1 we can derive a lower bound for $\left|\Lambda_{j}\right|$. Notice that $\max \left(j, n, 2\left|k_{j}\right|\right) \leqslant 2 n$, so that $W=\log (2 n)$. We choose $V_{1}=\frac{1}{2}$. The number $\alpha / \beta$ satisfies

$$
B x^{2}-\left(A^{2}-2 B\right) x+B=0
$$

hence $h(\alpha / \beta) \leqslant \frac{1}{2} \log B$. And $-\lambda / \mu$ satisfies

$$
E x^{2}-\left(2 E+\Delta G_{0}^{2}\right) x+E=0
$$

hence $h(-\lambda / \mu) \leqslant \frac{1}{2} \log E$. Thus $V_{2}=U_{2}^{+}, V_{3}=U_{3}^{+}$satisfy the requirements for Theorem 7.1. We find

$$
\begin{aligned}
\left|\Lambda_{j}\right| & >\exp \left\{-C_{4}^{\prime}\left(\log (2 n)+\log \left(2 e U_{3}^{+}\right)\right)\right\} \\
& =\exp \left\{-\left(C_{4}^{\prime} \log n+C_{5}^{\prime}\right)\right\} .
\end{aligned}
$$


Combining (7.3) and (7.4) we find $n<a+b \log n$, where

$$
\begin{aligned}
& a=\frac{2}{\log B}\left(\log \max \left(v, 2\left|G_{0} \mu \sqrt{\Delta}\right|\right)+\log \frac{\pi}{2|\mu|}+C_{5}^{\prime}\right), \\
& b=2 C_{4}^{\prime} / \log B .
\end{aligned}
$$

The result follows from Lemma 2.2 (Part I), since

$$
b=2 C_{4}^{\prime} / \log B=2^{78} 3^{6} \frac{\max (\pi, \log B)}{\log B} \max (\pi, \log E) \log \left(2 e U_{2}^{+}\right),
$$

which is certainly larger than $e^{2}$.

We now want to reduce the bound from Theorem 7.2. We do this by studying the diophantine inequality

$$
\left|\psi_{j}+n \phi+k_{j}\right|<v_{0} B^{-n / 2}
$$

where $\psi_{j}=j \psi$ and $v_{0}=\max \left(v, 2\left|G_{0} \mu \sqrt{\Delta}\right|\right) / 4|\mu|$. We have to distinguish between $\psi_{j}=0$ (the homogeneous case) and $\psi_{j} \neq 0$ (the inhomogeneous case).

7B. The Homogeneous Case. We first study the easier case $\psi_{j}=0$. We have the following algorithm. Let $N$ be an upper bound for the solutions of (7.5), for example the bound found in Theorem 7.2.

Algorithm B (reduces given upper bound for (7.5) in the case $\psi_{j}=0$ ).

Input: $\phi, B,|\mu|, v_{0}, N$.

Output: new, better bound $N^{*}$ for $n$.

(i) (initialization) Choose $n_{0} \geqslant 2 / \log B$ such that $B^{n_{0} / 2} / n_{0} \geqslant 2 v_{0} ; N_{0}:=[N]$; compute the continued fraction

$$
|\phi|=\left[0, a_{1}, a_{2}, \ldots, a_{l_{0}+1}, \ldots\right]
$$

and the denominators $q_{1}, \ldots, q_{l_{0}+1}$ of the convergents of $|\phi|$, with $l_{0}$ so large that $q_{l_{0}} \leqslant N_{0}<q_{l_{0}+1} ; i:=0$;

(ii) (compute new bound) $A_{i}:=\max \left(a_{1}, \ldots, a_{l_{i}+1}\right)$; compute the largest integer $N_{i+1}$ such that

$$
B^{N_{i+1} / 2} / N_{i+1} \leqslant v_{0}\left(A_{i}+2\right) ;
$$

and $l_{i+1}$ such that $q_{l_{i+1}} \leqslant N_{i+1}<q_{l_{i+1}+1}$;

(iii) (terminate loop)

$$
\text { if } n_{0} \leqslant N_{i+1}<N_{i} \quad \begin{aligned}
& \text { then } i:=i+1, \underline{\text { goto }} \text { (ii); } \\
& \text { else } N^{*}:=\max \left(n_{0}, N_{i+1}\right), \text { stop. }
\end{aligned}
$$

Lemma 7.3. Algorithm B terminates. Inequality (7.5) with $\psi_{j}=0$ has no solutions with $N^{*}<n<N$.

Proof. Termination is trivial, since all $N_{i}$ are integers. Notice that $B^{x / 2} / x$ is an increasing function for $x \geqslant 2 / \log B$. Hence, if $n \geqslant n_{0}$,

$$
|| \phi|-| k_{j}|/ n| \leqslant v_{0} B^{-n / 2} / n<1 / 2 n^{2} \text {. }
$$

It follows that $\left|k_{j}\right| / n$ is a convergent of $|\phi|$, say $\left|k_{j}\right| / n=p_{m} / q_{m}$. Then $q_{m} \leqslant n$, and, as is well known,

$$
|| \phi\left|-p_{m} / q_{m}\right|>1 /\left(a_{m+1}+2\right) q_{m}^{2}
$$


Suppose $n \leqslant N_{i}$ for some $i \geqslant 0$. Then $m \leqslant l_{i}$. Hence,

$$
B^{n / 2} / n \leqslant v_{0} n^{-2}|| \phi|-| k_{j}|/ n|^{-1}<v_{0}\left(a_{m+1}+2\right) \leqslant v_{0}\left(A_{m}+2\right) .
$$

It follows that if $N_{i+1} \geqslant n_{0}$, then $n \leqslant N_{i+1}$.

We notice that the above algorithm is similar to those of Cijsouw, Korlaar, and Tijdeman (appendix to Stroeker and Tijdeman [5]), and of D. C. Hunt and A. J. van der Poorten (unpublished manuscript).

7C. The Inhomogeneous Case. In the more complicated case $\psi_{j} \neq 0$, we use a technique due to H. Davenport (see Baker and Davenport [1, pp. 133-134]). Again, let $N$ be an upper bound for $n$.

Algorithm C (reduces upper bound for (7.5) in the case $\psi_{j} \neq 0$ ).

Input: $\quad \phi, \psi_{j}, B, v_{0}, N$.

Output: new, better upper bound $N^{*}$ for all but a finite number of explicitly given $n$.

(i) (initialization) $N_{0}:=[N]$; compute the continued fraction

$$
|\phi|=\left[0, a_{1}, \ldots, a_{l_{0}}, \ldots\right]
$$

and the convergents $p_{i} / q_{i}\left(i=1, \ldots, l_{0}\right)$, with $l_{0}$ so large that $q_{l_{0}}>4 N_{0}$ and $\left\|q_{l_{0}} \psi_{j}\right\|>2 N_{0} / q_{l_{0}}{ }^{*}$. (If such $l_{0}$ cannot be found within reasonable time, take $l_{0}$ so large that $\left.q_{l_{0}}>4 N_{0}\right) ; i:=0$;

(ii) (compute new bound)

if $\left\|q_{l_{i}} \psi_{j}\right\|>2 N_{i} / q_{l_{i}}$ then $N_{i+1}:=\left[2 \log \left(q_{l_{i}}^{2} v_{0} / N_{i}\right) / \log B\right]$;

$$
\begin{gathered}
\text { else compute } K \in \mathbb{Z} \text { with }\left|K-q_{l_{i}} \psi_{j}\right| \leqslant \frac{1}{2} ; \\
\text { compute } n_{0} \in \mathbb{Z}, 0 \leqslant n_{0}<q_{l_{i}} \text {, with } \\
K+n_{0} p_{l_{i}} \equiv 0\left(\bmod q_{l_{i}}\right), \\
\text { if } n=n_{0} \text { is a solution of }(7.5), \underline{\text { then }} \\
\text { print an appropriate message; } \\
N_{i+1}:=\left[2 \log \left(4 q_{l_{i}} v_{0}\right) / \log B\right] ;
\end{gathered}
$$

(iii) (terminate loop)

$$
\text { if } N_{i+1}<N_{i} \text { then } i:=i+1 \text {; }
$$

compute the minimal $l_{i}<l_{i-1}$ such that $q_{l_{i}}>4 N_{i}$ and $\left\|q_{l_{i}} \psi_{j}\right\|>2 N_{i} / q_{l_{i}}$ (If such $l_{i}$ does not exist, choose the minimal $l_{i}$ such that $\left.q_{l_{i}}>4 N_{i}\right)$;

goto (ii);

$$
\underline{\text { else }} N^{*}:=N_{i} \text {, stop. }
$$

LEMMA 7.4. Algorithm C terminates. Inequality (7.5) with $\psi_{j} \neq 0$ has for $N^{*}<n$ $<N$ only the finitely many solutions found by the algorithm.

Proof. It is clear that the algorithm terminates. Suppose that $n \leqslant N_{i}$ for some $i \geqslant 0$. Then if $\left\|q_{l_{i}} \psi_{j}\right\|>2 N_{i} / q_{l_{i}}$, we have

$$
\begin{aligned}
\left\|q_{l_{i}} \psi_{j}\right\| & =\left\|q_{l_{i}}\left(\psi_{j}+n \phi+k_{j}\right)-n \phi q_{l_{i}}\right\| \\
& \leqslant q_{l_{i}}\left|\psi_{j}+n \phi+k_{j}\right|+n / q_{l_{i}} \leqslant q_{l_{i}} v_{0} B^{-n / 2}+N_{i} / q_{l_{i}} .
\end{aligned}
$$

* $\|\cdot\|$ denotes the distance to the nearest integer. 
It follows that $n \leqslant N_{i+1}$. If $\left\|q_{l_{i}} \psi_{j}\right\| \leqslant 2 N_{i} / q_{l_{i}}$, then

$$
\begin{aligned}
\left|K+n p_{l_{i}}+k_{j} q_{l_{i}}\right| & \leqslant\left|K-q_{l_{i}} \psi_{j}\right|+q_{l_{l}}\left|\psi_{j}+n \phi+k_{j}\right|+n\left|p_{l_{i}}-q_{l_{i}} \phi\right| \\
& \leqslant \frac{1}{2}+q_{l_{i}} v_{0} B^{-n / 2}+N_{i} / q_{l_{i}}<\frac{3}{4}+q_{l_{i}} v_{0} B^{-n / 2} .
\end{aligned}
$$

Suppose that $q_{l_{i}} v_{0} B^{-n / 2} \leqslant \frac{1}{4}$. Then $K+n p_{l_{i}}+k_{j} q_{l_{i}}=0$, since it is an integer. By $\left(p_{l_{i}}, q_{l_{i}}\right)=1$ it follows that $n \equiv n_{0}\left(\bmod q_{l_{i}}\right)$. Since $q_{l_{i}}>N_{i}, n=n_{0}$ is the only possibility. Suppose next that $q_{l} v_{0} B^{-n / 2}>\frac{1}{4}$. Then $n \leqslant N_{i+1}$ follows immediately.

We remark that in practice one almost always finds an $l_{i}$ such that $\left\|q_{l_{i}} \psi_{j}\right\|>$ $2 N_{i} / q_{l_{i}}$, if $N_{i}$ is large enough.

\section{How to Solve (1.1).}

8A. Bounds for the Solutions. Combining the results from the $p$-adic and the complex Gelfond-Baker theory (Lemma 3.2 and Theorem 7.2), we now derive upper bounds for the solutions of (1.1) with $\Delta<0$.

Theorem 8.1. Put $C_{1}=\max _{1 \leqslant i \leqslant t}\left(C_{1, i}\right)$ and $P=p_{1} \cdots p_{t}$. Further, put

$$
\begin{gathered}
C_{7}=\max \left\{C_{6}+\frac{4}{\log B} \log \left(2\left|G_{0} \mu \sqrt{\Delta}\right|\right),\right. \\
\left.8\left(\left(C_{6}+\frac{4 \log |w|}{\log B}\right)^{1 / 3}+\left(\frac{4 C_{1} \log P}{\log B}\right)^{1 / 3} \log \left(\frac{108 C_{1} \log P}{\log B}\right)\right)^{3}\right\}, \\
C_{8, i}=C_{1, i}\left(\log C_{7}\right)^{3} \quad(i=1, \ldots, t) .
\end{gathered}
$$

Then all solutions of (1.1) satisfy

$$
n<C_{7}, \quad m_{i}<C_{8, i} \quad(i=1, \ldots, t) .
$$

Proof. From Lemma 3.2 and Theorem 7.2 with $v=|w| p_{1}^{m_{1}} \cdots p_{t}^{m_{t}}$, we see that

$$
n<C_{6}+\frac{4}{\log B} \log \left(2\left|G_{0} \mu \sqrt{\Delta}\right|\right),
$$

or

$$
n<C_{6}+\frac{4 \log |w|}{\log B}+\frac{4 C_{1} \log P}{\log B}(\log n)^{3} .
$$

The result now follows from Lemma 2.2 if $4 C_{1} \log P / \log B>\left(e^{2} / 3\right)^{3}$. This is certainly true.

8B. The Algorithm. We present an algorithm to reduce upper bounds for the solutions of Eq. (1.1). The idea is to apply alternatingly algorithms A and one of B and C. Let $N$ be an upper bound for $n$, for example $N=C_{7}$.

Algorithm D (reduces upper bounds for the solutions of (1.1)).

Input: $\alpha, \beta, \lambda, \mu, w, p_{1}, \ldots, p_{t}, N$.

Output: new, better bounds $N^{*}, M_{i}$ for $n$ and $m_{i}(i=1, \ldots, t)$.

(i) (initialization) $N_{0}:=[N] ; j:=1$;

$$
\begin{aligned}
& g_{i}:=\operatorname{ord}_{p_{i}}(\lambda)+\operatorname{ord}_{p_{i}}\left(\log _{p_{i}}(\alpha / \beta)\right) \\
& h_{i}:=\operatorname{ord}_{p_{i}}(\lambda)+\left\{\begin{array}{cc}
3 / 2 & \text { if } p_{i}=2 \\
1 & \text { if } p_{i}=3 \\
1 / 2 & \text { if } p_{i} \geqslant 5
\end{array}\right\} \quad(i=1, \ldots, t) ;
\end{aligned}
$$


(ii) (computation of the $\theta_{i}$ 's, $\phi$ and $\psi$ )

compute for $i=1, \ldots, t$ the first $r_{i} p_{i}$-adic digits of

$$
\theta_{i}=-\log _{p_{i}}(-\lambda / \mu) / \log _{p_{i}}(\alpha / \beta)=\sum_{l=0}^{\infty} u_{i, l} p_{i}^{l},
$$

where $r_{i}$ is so large that $p_{i}^{r_{1}} \geqslant N_{0}$ and $u_{i, r_{i}} \neq 0 ;$ compute $\psi=\log (-\lambda / \mu) / 2 \pi i$, and the continued fraction

$$
|\phi|=\left|\frac{1}{2 \pi i} \log (\alpha / \beta)\right|=\left[0, a_{1}, \ldots, a_{l_{0}}, \ldots\right]
$$

with the convergents $p_{i} / q_{i}\left(i=1, \ldots, l_{0}\right)$, where $l_{0}$ is so large that $q_{l_{0}-1} \leqslant N_{0}$ $<q_{l_{0}}$ if $\psi=0 ; q_{l_{0}}>4 N_{0}$ and $\left\|q_{l_{0}} \psi\right\|>2 N_{0} / q_{l_{0}}$ if $\psi \neq 0$ and such $l_{0}$ can be found in a reasonable amount of time, $q_{l_{0}}>4 N_{0}$ otherwise.

(iii) (one step of Algorithm A)

$M_{i, j}:=\max \left(h_{i}, g_{i}+\min \left\{s \in \mathbb{Z}: s \geqslant 0\right.\right.$ and $p_{i}^{s} \geqslant N_{j-1}$ and $\left.\left.u_{i, s} \neq 0\right\}\right)(i=$ $1, \ldots, t)$;

(iv) (one step of Algorithm $\mathrm{B}$ or $\mathrm{C}$ )

if $\psi=0$ then $A:=\max \left(a_{1}, \ldots, a_{l,-1}\right) ; v:=|w| p_{1}^{M_{1,1}} \cdots p_{t}^{M_{t, j}}$;

choose $n_{0} \geqslant 2 / \log B$ such that $B^{n_{0} / 2} / n_{0} \geqslant v / 2|\mu|$;

compute the largest integer $N_{j}$ such that $B^{N_{1} / 2} / N_{j} \leqslant$

$(A+2) v / 4|\mu|$;

$N_{j}:=\max \left(n_{0}, N_{j}\right)$;

if $N_{j}<N_{j-1}$ then compute $l_{j}$ such that

$q_{l,-1} \leqslant N_{j}<q_{l}$;

$j:=j+1$; goto (iii);

$\underline{\text { else if }}\left\|q_{l,}, \psi\right\|>2 N_{j-1} / q_{l, \text {, }}$

then $N_{j}:=\left[2 \log \left(q_{l,-1}^{2} v / 4|\mu| N_{j-1}\right) / \log B\right]$;

else compute $K \in \mathbb{Z}$ with $\left|K-q_{l_{1,1}} \psi\right| \leqslant \frac{1}{2}$;

compute $n_{0} \in \mathbb{Z}, 0 \leqslant n_{0}<q_{l, 1}$,

with $K+n_{0} p_{l_{1-1}} \equiv 0\left(\bmod q_{l_{1,1}}\right)$;

if $n=n_{0}$ is a solution of (1.1)

then print an appropriate message;

$\left.N_{j}: \overline{=[2} \log \left(q_{l_{i-1}} v /|\mu|\right) / \log B\right]$;

if $N_{j}<N_{j-1}$ then compute the minimal $l_{j}<l_{j-1}$ such that

$q_{l,}>4 N_{j}$ and $\left\|q_{l}, \psi\right\|>2 N_{j} / q_{l,}$ (if such $l_{j}$ does not exist, choose the minimal $l_{j}$ such that $\left.q_{l,}>4 N_{j}\right)$

$j:=j+1$; goto (iii);

(v) (termination) $N^{*}:=N_{j-1} ; M_{i}:=M_{i, j}(i=1, \ldots, t)$; stop.

THEOREM 8.2. Algorithm D terminates. Equation (1.1) has no solutions with $N^{*}<n<N$ and $m_{i}>M_{i}(i=1, \ldots, t)$, apart from those spotted by the algorithm.

Proof. Clear, from the proofs of Lemmas 7.3 and 7.4.

8C. An Example. Let $A=1, B=2, G_{0}=2, G_{1}=3$, then $\Delta=-7, \alpha=$ $(1+\sqrt{-7}) / 2, \lambda=(2+\sqrt{-7}) / \sqrt{-7}$. Let $w= \pm 1, p_{1}=3, p_{2}=7$. We have with $n_{0}=2: C_{1}<6.40 \times 10^{16}, C_{6}<9.14 \times 10^{29}, C_{7}<7.42 \times 10^{30}, C_{8}<2.30 \times 10^{22}$. 
Further, $g_{1}=1, g_{2}=0, h_{1}=1, h_{2}=0$. Let $N_{0}=7.42 \times 10^{30}$. We have

$$
\begin{aligned}
& \phi=\log (\alpha / \beta) / 2 \pi i=(\pi-\arctan (\sqrt{7} / 3)) / 2 \pi \\
&= {[0,2, \quad 1,1,2,16, \quad 6,1,2,2,13,} \\
& 1, \quad 1,3,1,1, \quad 2,1,2,1,1, \\
& 1, \quad 1,1,9,2, \quad 1,2,1,7,1, \\
& 6,269,4,3,1, \quad 1,50,2,1,6, \\
& 1, \quad 1,2,1,1, \quad 7,1,61,1,12, \\
&3, \quad 7,4,7,3,121,1,21,2,1,7, \ldots], \\
& \psi=\log (-\lambda / \mu) / 2 \pi i=(\pi-\arctan (4 \sqrt{7} / 3)) / 2 \pi \\
&=0.2939628336996454026789566605200190806203 \ldots, \\
& \theta_{1}=0.2001012210000110210200211002220222012021 \\
& 1002020202211020012101000010021110020122 \\
& 111112220221021022122200 \ldots, \\
& \theta_{2}=0.3254212042435613402061561134521011633152 \\
& 25336450441125455033 \ldots
\end{aligned}
$$

Now, $M_{1,1}=67, M_{2,1}=37$; we choose $l_{0}=61$, since

$$
q_{61}=142511833114244361193755123881743>4 N_{0},
$$

and $\left\|q_{61} \psi\right\|=0.24487 \ldots>2 N_{0} / q_{61}=0.104 \ldots$ So we find $N_{1}=637$. Next, $M_{1,2}$ $=7, \quad M_{2,2}=4 ;$ we choose $l_{1}=9$, since $q_{9}=10102>4 \times 637$, and $\left\|q_{9} \psi\right\|=$ $0.38745 \ldots>2 \times 637 / 10102$. So we find $N_{2}=74$. Next, $M_{1,3}=6, M_{2,3}=3$; we choose $l_{2}=6$, since $q_{6}=1291>4 \times 74$, and $\left\|q_{6} \psi\right\|=0.49398 \ldots>2 \times 74 / 1291$. So we find $N_{3}=60$. In the next step we find no improvement. Hence $n \leqslant 60$, $m_{1} \leqslant 6, m_{2} \leqslant 3$. It is a matter of straightforward computation to check that there are the following 6 solutions of $G_{n}= \pm 3^{m_{1}} 7^{m_{2}}: G_{1}=3, G_{2}=-1, G_{3}=-7$, $G_{5}=9, G_{7}=1, G_{17}=441$.

9. A Mixed Quadratic-Exponential Equation. In this section, we give an application of the preceding algorithm to the following diophantine equation. Let

$$
\Phi(X, Y)=a X^{2}+b X Y+c Y^{2}
$$

be a quadratic form with integral coefficients, such that $D=b^{2}-4 a c<0$. Let $q$, $v, w$ be nonzero integers, and $p_{1}, \ldots, p_{t}$ prime numbers. Consider the equation

$$
\left\{\begin{array}{l}
\Phi(X, Y)=v q^{n} \\
Y=w p_{1}^{m_{1}} \cdots p_{t}^{m_{t}}
\end{array}\right.
$$

in integers $X, n \geqslant 0, m_{i} \geqslant 0(i=1, \ldots, t)$.

Let $\beta, \bar{\beta}$ be the roots of $\Phi(x, 1)$. Let $h$ be the class number of $\mathbb{Q}(\sqrt{D})$. There exists a $\pi \in \mathbf{Q}(\sqrt{D})$ such that we have the principal ideal equation $(\pi)(\bar{\pi})=\left(q^{h}\right)$. Put $n=n_{1}+h n_{2}$, with $0 \leqslant n_{1}<h$. Then $\Phi(X, Y)=v q^{n}$ is equivalent to finitely many ideal equations

$$
(a X-a \beta Y)(a X-a \bar{\beta} Y)=(\sigma)(\bar{\sigma})(\pi)^{n_{2}}(\bar{\pi})^{n_{2}},
$$


with $(\sigma)(\bar{\sigma})=\left(a v q^{n_{1}}\right)$. Hence we have the equations (in algebraic numbers)

$$
\left\{\begin{array} { l } 
{ a X - a \beta Y = \gamma \pi ^ { n _ { 2 } } , } \\
{ a X - a \overline { \beta } Y = \overline { \gamma } \overline { \pi } ^ { n _ { 2 } } , }
\end{array} \quad \left\{\begin{array}{l}
a X-a \beta Y=\gamma \bar{\pi}^{n_{2}}, \\
a X-a \bar{\beta} Y=\bar{\gamma} \pi^{n_{2}},
\end{array}\right.\right.
$$

where $\gamma$ is composed of units, common divisors of $a X-a \beta Y, a X-a \bar{\beta} Y$, and $\sigma$. Notice that there are only finitely many choices for $\gamma$ possible. Thus, (9.1) is equivalent to a finite number of equations

$$
a(\bar{\beta}-\beta) w p_{1}^{m_{1}} \cdots p_{t}^{m_{t}}=\gamma \pi^{n_{2}}-\bar{\gamma} \bar{\pi}^{n_{2}},
$$

or, if we put $\lambda=\gamma / a(\bar{\beta}-\beta)$ and $G_{n_{2}}=\lambda \pi^{n_{2}}+\bar{\lambda} \bar{\pi}^{n_{2}}$,

$$
G_{n_{2}}=w p_{1}^{m_{1}} \cdots p_{t}^{m_{t}} \text {. }
$$

Here $\left\{G_{n_{2}}\right\}_{n_{2}=0}^{\infty}$ is a recurrence sequence with negative discriminant. So (9.2) is of type (1.1), and it can thus be solved by the method presented in Sections 7 and 8.

Before giving an example, we remark that Eq. (9.1) with $D>0$ is not solvable with our method. This is due to the fact that in $\mathbb{Q}(\sqrt{D})$ with $D>0$ there are infinitely many units, hence infinitely many possibilities for $\gamma$. Another generalization of Eq. (9.1) is to replace $q^{n}$ by $q_{1}^{n_{1}} \cdots q_{s}^{n_{s}}$. This problem is also not solvable by our method, since it does not lead to a binary recurrence sequence if $s \geqslant 2$. It seems that these problems can be solved by using multi-dimensional approximation techniques. This is the subject of further investigations by the author.

We finally present an example.

THEOREM 9.1. The equation

$$
X^{2}-3^{m_{1}} 7^{m_{2}} X+2\left(3^{m_{1}} 7^{m_{2}}\right)^{2}=11 \cdot 2^{n}
$$

in integers $X, n \geqslant 0, m_{1} \geqslant 0, m_{2} \geqslant 0$ has only the following solutions:

\begin{tabular}{rrrrrrrrrr}
$n$ & $m_{1}$ & $m_{2}$ & \multicolumn{2}{c|}{$X$} & $n$ & $m_{1}$ & $m_{2}$ & \multicolumn{2}{c}{$X$} \\
\hline 1 & 1 & 0 & -1, & 4 & 5 & 2 & 0 & -10, & 19 \\
1 & 0 & 0 & -4, & 5 & 6 & 0 & 0 & -26, & 27 \\
2 & 0 & 0 & -6, & 7 & 7 & 0 & 0 & -37, & 38 \\
3 & 0 & 1 & 2, & 5 & 7 & 3 & 0 & 2, & 25 \\
3 & 1 & 0 & -7, & 10 & 11 & 1 & 1 & -137, & 158 \\
4 & 0 & 1 & -6, & 13 & 17 & 2 & 2 & -829, & 1270
\end{tabular}

Sketch of Proof. Put $\beta=(1+\sqrt{-7}) / 2$. Then

$$
X^{2}-X Y+2 Y^{2}=(X-\beta Y)(X-\bar{\beta} Y) \text {. }
$$

Notice that $\mathbb{Q}(\sqrt{-7})$ has class number 1 , and that

$$
2=(1+\sqrt{-7}) / 2 \times(1-\sqrt{-7}) / 2, \quad 11=(2+\sqrt{-7})(2-\sqrt{-7}) .
$$

Suppose $\gamma \mid X-\beta Y$ and $\gamma \mid X-\bar{\beta} Y$. Then $\gamma \mid(\bar{\beta}-\beta) Y=-\sqrt{-7} 3^{m_{1}} 7^{m_{2}}$. On the other hand, $\gamma \mid 11 \cdot 2^{n}$. It follows that $\gamma= \pm 1$; hence $X-\beta Y$ and $X-\bar{\beta} Y$ are coprime. Thus we have two possibilities:

$$
\begin{aligned}
& X-\beta Y= \pm(2 \pm \sqrt{-7})\left(\frac{1 \pm \sqrt{-7}}{2}\right)^{n}, \\
& X-\beta Y= \pm(2 \mp \sqrt{-7})\left(\frac{1 \pm \sqrt{-7}}{2}\right)^{n},
\end{aligned}
$$


in each equation the 2 nd and 3 rd \pm being independent. Hence, we have to solve

$$
G_{n}^{(j)}=\lambda^{(j)} \beta^{n}+\bar{\lambda}^{(j)} \bar{\beta}^{n}=3^{m_{1}} 7^{m_{2}} \quad(j=1,2),
$$

with $G_{n+1}^{(j)}=G_{n}^{(j)}-2 G_{n-1}^{(j)}(j=1,2)$ and $\lambda^{(1)}=\bar{\lambda}^{(2)}=(2+\sqrt{-7}) / \sqrt{-7}$, so that $G_{0}^{(1)}=G_{0}^{(2)}=1, G_{1}^{(1)}=3, G_{1}^{(2)}=-1$. Notice that $\theta_{i}^{(1)}=-\theta_{i}^{(2)}(i=1,2)$, and $\psi^{(1)}=$ $-\psi^{(2)}$. For $j=1$ we solved (9.3) in the example of Subsection $8 \mathrm{C}$. We leave it to the reader to solve $(9.3)$ for $j=2$; this can be done with the numerical data given in Subsection $8 \mathrm{C}$.

Acknowledgments. The author wishes to thank F. Beukers, A. Pethö and R. Tijdeman for their comments. He was supported by the Netherlands Foundation for Mathematics (SMC) with financial aid from the Netherlands Organization for the Advancement of Pure Research (ZWO).

Mathematisch Instituut R. U. Leiden

Postbus 9512

2300 RA Leiden

The Netherlands

1. A. Baker \& H. Davenport, "The equations $3 x^{2}-2=y^{2}$ and $8 x^{2}-7=z^{2}$," Quart. J. Math. Oxford Ser. (2), v. 20, 1969, pp. 129-137.

2. P. Kiss, “Zero terms in second order linear recurrences," Math. Sem. Notes Kobe Univ., v. 7, 1979, pp. $145-152$.

3. K. Mahler, "Eine arithmetische Eigenschaft der rekurrierenden Reihen," Mathematika B (Leiden), v. 3, 1934, pp. 153-156.

4. A. Pethö \& B. M. M. DE Weger, "Products of prime powers in binary recurrence sequences. I," Math. Comp., v. 47, 1986, pp. 713-727.

5. R. J. Stroeker \& R. Tijdeman, "Diophantine equations," in Computational Methods in Number Theory (H. W. Lenstra, Jr. and R. Tijdeman, eds.), MC Tract 155, Amsterdam, 1982, pp. 321-369.

6. M. WAldschmid, “A lower bound for linear forms in logarithms," Acta Arith., v. 37, 1980, pp. $257-283$. 